\title{
Ecopharmacognosy: Exploring the Chemical and Biological Potential of Nature for Human Health
}

\author{
Geoffrey A. Cordell \\ Natural Products Inc., Evanston, Illinois 60203, USA \\ Author correspondency: \\ pharmacog@gmail.com
}

\begin{abstract}
"Why didn't they develop natural product drugs in a sustainable manner at the beginning of this century?" In 2035 , when about 10.0 billion will inhabit Earth, will this be our legacy as the world contemplates the costs and availability of synthetic and gene-based products for primary health care? Acknowledging the recent history of the relationship between humankind and the Earth, it is essential that the health care issues being left for our descendants be considered in terms of resources. For most people in the world, there are two vast health care "gaps", access to quality drugs and the development of drugs for major global and local diseases. Consequently for all of these people, plants, in their various forms, remain a primary source of health care. In the developed countries, natural products derived from plants assume a relatively minor role in health care, as prescription and over-the-counter products, even with the widespread use of phytotherapeutical preparations. Significantly, pharmaceutical companies have retrenched substantially in their disease areas of focus. These research areas do not include the prevalent diseases of the middle- and lower-income countries, and important diseases of the developed world, such as drug resistance. What then is the vision for natural product research to maintain the choices of drug discovery and pharmaceutical development for future generations? In this discussion some facets of how natural products must be involved globally, in a sustainable manner, for improving health care will be examined within the framework of the new term "ecopharmacognosy", which invokes sustainability as the basis for research on biologically active natural products. Access to the biome, the acquisition, analysis and dissemination of plant knowledge, natural product structure diversification, biotechnology development, strategies for natural product drug discovery, and aspects of multitarget therapy and synergy research will be discussed. Options for the future will be presented which may be significant as countries decide how to develop approaches to relieve their own disease burden, and the needs of their population for improved access to medicinal agents.
\end{abstract}

Keywords: natural products, ecopharmacognosy, sustainable medicines, biotechnology, structure diversification, rain forest resources, strategic implications

\section{Introduction}

In Lewis Carroll's famous book "Alice in Wonderland; Through the Looking Glass", it is the Mad Hatter who has the perspicacity to say to Alice "We are all mad here". In many ways, given the recent and ongoing relationship between humankind and the resources of the Earth, it is we who are living in a world of madness, where resources are squandered now, for instant benefit, with little consideration given for the resource needs of the generations to follow. And it we who, in spite of numerous warnings and international meetings and declarations, still cannot turn and say clearly that what we are doing to the planet and its resources is indeed "madness", and must change if the human race is to survive and thrive. To borrow a phrase, "We are killing the planet that heals us".

The core madness in the scenario is this: a dramatically increasing population, mostly in the middleand low-income countries, an alarming decrease in the acreage of tropical rain forests, and an expanding dependence on those resources and others for food, shelter, and health care. When, as we have seen, most of the world relies on medicinal plants for their health care, and up to $85 \%$ of those plants are harvested in a nonrenewable manner, it is clear to see that new strategies are needed to assure access to medicinal agents in the future
(1-12). The fourth factor, oil, remains a mysterious unknown, an enigma with respect to long-term accessibility. Optimistic estimates of the current oil resources, although accurate data are basically nonavailable for financial and security reasons, would suggest that they may be viable for the next $40-50$ years. However, population is increasing at a faster rate than oil production. So what will be the status of the Earth's resources at that time, say in 2060, when renewable, moderate cost, effective, alternative sources for synthetic medicinal agents will be needed?

Enzymes, isolated or in intact systems, must be deployed as an essential component of industrial drug synthesis to reduce the dependency on non-renewable resources. The July, 2011 issue of Chemical Reviews was devoted to a series of eleven reviews of different types of enzymes deployed for selective synthetic transformations, several related to drug processes. The use of renewable, multifunctional enzyme systems which can transpose a compound through to a final product with minimal "chemical" reagent involvement may be an important facet of the production of synthetic drugs within ten years. Considerations of the use of inexpensive renewable reagents may also drive the selection of both production processes and compounds evaluated for their drug potential and eventual marketing. Also to be developed further is the potential for the use of 
vegetables as chemical reagents for selected enantioselective processes, where intact materials, not isolated enzymes are utilized (13). However, the need for natural products, as quality traditional medicines, and as sources for new medicinal agents will remain. This brief discussion is about some of those opportunities.

As natural product scientists, one of our obligations is to initiate the discussion of how to investigate natural resources for health care, and encourage others to see different perspectives of the overall picture. Our role is also to examine, define, and articulate the niche of natural products in the future of the population of Earth, and particularly the anticipated role of natural products in global health care. It is also important that we, as the content experts, have a scientific role in developing those future plans.

The global population is now at 7.31 billion (April 2013), and is projected to rise to 10 billion by about 2035 . The causes and the implications for such a dramatic population explosion are philosophical and religious, which are considerations beyond the scope of this short article, but upon which the fate of human existence hinges $(14,15)$. As one small, yet significant aspect of this huge discussion, some of the many integrated aspects of natural products in health care, we will review some possible relationships between natural products and drug discovery, and look one again at the important question: "What is the future for natural products in global health?" The author has written numerous articles on this area in the past 25 years $(1-6,16-24)$, with several of the more recent articles focusing on two major issues, the concept of medicines as a sustainable commodity (4-12), and enhancing the quality control of traditional medicines in global health (6-12,23-26).

On a daily basis, we often forget to acknowledge with gratefulness the vast contributions that plants, their extracts, and the various products derived from them, make to human health and well-being: i) as foodstuffs, ii) as flavoring agents and spices, iii) as perfumes and cosmetics, and iv) as pharmaceutical and biological agents. These categories are not mutually exclusive, for it is apparent that there is significant overlap between foods, spices, cosmetics, and biological (medicinal) agents.

It was estimated over 20 years ago that there are more than 120 compounds from over 90 different plant materials which are used on a global basis as prescription products (27), and this number may well have risen since then (28-32). Butler, for example, lists 34 natural product based drugs introduced in the period 1998-2007 (31), and Harvey lists 108 plant-derived compounds in various development phases (32). From a commercial perspective, world-wide sales for plant-based pharmaceutical agents in 2002 were estimated at US $\$ 30$ billion (33). In addition, there are also thousands of products, compounds, plant extracts and plant materials, some sanctioned as prescription products, others bought over-the-counter, or in a market place, or through a local medicine man or woman, which are recommended for patient healing in various parts of the world. For the vast majority of the world, approximately 4.5 billion people, these plant resources are their primary source of health care (34).

There are many "gaps" in global health care, and per capita government investment in health care in a particular country (see Table 1 for some examples), is one of the more staggering examples between the highincome and the low-income countries. That aspect of health care concerned with medicinal agents for patient treatment, pharmacy, is no exception. For example, there is a major regulatory "gap" between the prescription products of the North and the products sold in a marketplace as part of a traditional medicine system in a middle- or low-income country. One is highly regulated, costing perhaps $\$ 1.3$ billion to develop a product and support continuing post-marketing surveillance (35). Whereas the traditional medicine is almost completely unregulated; in many countries, a plant material appears in the street market directly from the field, just as it did 4000 years ago, and there are many variations of regulation in this continuum of extremes.

The UN charter of 1948 clearly indicates that all humans have the universal right to health care. But what is being done by the organizations of the $\mathrm{UN}$ to achieve this? What does that universal right mean in terms of the quality control of traditional medicines or for drug discovery for global and local diseases? In this regard, we must not be limited to thinking that drug discovery involves only single compounds. In an era of systems biology and network pharmacology, it is clear that the period for thinking of drug discoveries as "magic bullets" is long gone.

Table 1. Government Expenditures on Health per Capita (2009) in US $\$$.

\begin{tabular}{ll}
\hline Country & US \$ \\
\hline United States & 7960 \\
Norway & 5383 \\
Australia & 3484 \\
United Kingdom & 3438 \\
Republic of Korea & 1879 \\
Costa Rica & 1155 \\
Turkey & 957 \\
South Africa & 930 \\
Brazil & 921 \\
Iran & 728 \\
Peru & 466 \\
China & 347 \\
Vietnam & 204 \\
Philippines & 133 \\
India & 124 \\
Nigeria & 136 \\
Indonesia & 100 \\
Myanmar & 36 \\
\hline
\end{tabular}

Source:http://en.wikipedia.org/wiki/List_of_countries_by_total_health_ex penditure_(PPP)_per_capita

In terms of plant-based health care, research is drastically under-funded at the global and national levels in terms of personnel, programs, products, and outcomes. 
The truth is that, as a moral and ethical issue, it is a shameful international embarrassment, and requires a complete reassessment of strategies, programs, and outcomes from the very top of the UN through all of the various agencies involved. One group, Médecins Sans Frontieres, justifiably, has called for a complete overall of the whole drug discovery, development and access system for the middle and lower-income areas of the world given the history of drug innovation for the South in the past thirty years (35).

Patients world-wide who acquire plant-based products, either through prescription or as a street medicine, in their various forms, are rightfully demanding the same assurances of safety, efficacy, and consistency as a patient taking a drug from a pharmaceutical company. The "gap", of course, is who is standing behind those assurances? Is it a government, a pharmaceutical company, a traditional medicine provider? If no-one is making (or can make) those fundamental assurances, based on science, what does that mean for the overall health care of a nation? As the wise man once said, if you want the answer.... "follow the money."

Although very basic, this is merely one of many health care "gaps" that exist in the world at this time; another gaggle of "gaps" concern the question of access. In this sense, the term is applied in four ways. The first facet is what are the prevalent diseases in a country for which drugs are not available at all? The second is what are the diseases for which drugs are available globally, but which are either too costly, or not available, locally? The third facet is what are the diseases for which there is resistance to existing treatments? The final facet of access relates to the source of medicinal plant materials, and the long term stability of a disappearing forest as a sustainable source of medicinal agents. These facets, individually or collectively, can form the basis for rationalizing the need for targeted new drug discovery programs, and for placing that rationalization in a conservation and sustainable development framework. Access to health care, and to medications in particular, is consequently a global concern for all, with the exception of the very wealthy.

Another of the medicine-related "gaps" in health care relates to two questions: what are global health care needs? And what is contemporary drug discovery in the pharmaceutical industry targeting? The "gap" between these two responses is vast and expanding. The global health needs for over 1 billion people on Earth are treatments for: malaria, HIV-AIDS, tuberculosis, hepatitis $\mathrm{C}$, diarrheal diseases, ascariasis, leishmaniasis, schistosomiasis, trypanosomiasis, dengue fever, leprosy, rabies, yaws, and necatoriasis. Contemporary drug discovery areas in the major pharmaceutical companies have been winnowed to: antivirals, oncology, metabolism, central nervous system ailments, and inflammatory diseases (36). More recently, one major company, AstraZeneca, has winnowed its research portfolio to three areas: inflammatory and autoimmune diseases, cardiovascular and metabolic diseases, and oncology (37). The logic behind these strategies is that drug companies will make money when they produce drugs which: i) will not encounter a resistance issue, as occurs with antibiotics, ii) are taken on a chronic basis, and iii) are palliative and not curative. The global health disease burden is not their concern. It is really that simple.

For individual countries, and for regional associations of countries, such as ASEAN, many questions must be asked in terms of the present and the future health care needs. There are limited precedents available, but recall that it may take at least fifteen years to move from bench to marketplace, if the strategy involves the development of single agent drugs. There are alternatives which also should be considered, and which, depending on the particular country situation, can serve either as interim steps for enhancing health care, or as longer term solutions. Clearly though, not every country will have the human capacity, the infrastructure, and the financial resources to muster a drug discovery effort based on the study of their natural resources. Even those countries who do embark on that endeavor will need to clarify whether all of the needed expertise is available in country, or whether other regional or global resources need to be accessed.

From a discovery perspective, countries have two main natural resources at their disposal, their natural flora and the indigenous knowledge, which may be within one or several traditional systems of medicine. These resources offer a unique basis with which to develop a strategy or a series of strategies for drug discovery. In this instance, the emphasis here is on a government ministry, or most likely a group of ministries (science and technology, health, education, agriculture, commerce), and local (not the major) pharmaceutical companies, coming together to serve as a catalyst to address this aspect of health care. Initiatives such as the discovery of drugs or the enhancement of traditional medicines to meet societal health needs cannot begin at the local level, but must come "top down", where government is both "calling the shots" through establishing consultative groups, and at the same time providing the highly targeted resources for addressing long-term needs. All parties, government regulators and funding sources, botanical, chemical and biological scientists, agronomists, industrialists, and academics need to come together to address these basic health care issues as a coordinated and cooperative venture for the health of their population.

As we consider how to potentiate the available natural resources for health care, it is also important to examine the historical background to the interest in the tropical forests. It is not adequate to state that because plants have yielded drugs in the past they will do so in the future. Neither is it appropriate to conclude that because a particular plant has a long history of use as a traditional medicine that it should be approved as a drug. Many other considerations come into play, including the need to place such assertions in science; the evidence-based approach.

So what is the goal, and what are the strategies, of exploring the rain forest for medicinal plants? Are there 
clear and rational reasons which can adequately justify the investment? If so, what is the nature (breadth, depth, duration) of such an interest? What if the discovery of either an effective plant extract or an effective compound is made? What are the issues with respect to next stage development, and how will further development, if warranted, be supported financially? Where and how does the patenting of inventions impact the development process? What are the local implications in terms of agricultural or infrastructure development?

Potentially, unraveling the human genome provides an opportunity for the development of assays related to numerous disease-related, new drug targets, once those correlations have been determined. Because of the ethnomedical reputation of tens of thousands (perhaps 8$10 \%$ ) of terrestrial plants (4), once a prioritized acquisition and screening schedule is achieved, very specific opportunities for the selective evaluation of the effectiveness and potential for further development of traditional medicines may be achieved.

Strategically though, for the wider evaluation of plant materials, and for more rapid and effective quality control assessment of traditional medicines an important paradigm needs to be reversed (9-12). As a modality for conducting preliminary screening programs for the biological activity of plant extracts, bringing plant materials from the collection site to the laboratory for extraction and bioassay is an exceptionally inefficient approach. Depending where an "active" line is drawn, but probably around the $2-4 \%$ level for most programs, the extracts of $96-98 \%$ of the collected plant materials are "inactive" in any particular biological assay. While those materials (and their parent plants) can be retained and stored as an asset to the program, and subsequently reassessed against new target assays in the future, the fact remains that there is significant effort in collection, drying, grinding, and extraction which is wasted. Consequently, there is an urgent need for the development of bioassay systems, preferably genomebased, which can be performed, at collection site locations, directly on plant extracts prepared in the field using micro-extraction technology to remove unwanted plant constituents. Further local plant collection would then focus on those plant extracts which have a demonstrated biological activity. At the same time, the frequent failure of plant extracts to reconfirm biological activity on recollection will be reduced.

Who is potentially interested in exploring the rain forest biodiversity for bioactive products? Several types of companies have a financial interest in plant-based natural products and extracts. In spite of the success of plant-based pharmaceuticals, most of the large pharmaceutical companies have terminated those aspects of their drug discovery programs which are based on plant-derived natural products. The prime reasons for this have been discussed elsewhere $(1,3,4,9-12,20)$. As complex matrices, plant extracts provide a significant challenge for the bioactivity-directed isolation of the active principle(s). In addition, this deconvolution step may yield an undesirable known, rather than a novel, active constituent. The second consideration is that the recollection of a plant may be a time-consuming process, require extensive (re-)negotiations related to access, and may not be biologically active. Yet another consideration is the ability to provide an adequate supply of a compound for additional biological evaluation in a timely and reproducible manner.

For some biotechnology companies, the use of tissue culture systems in order to examine the ability of cell-free plant systems to produce metabolites which are not present in field-grown plants has proved important, and has provided an opportunity to study plant secondary metabolic enzyme systems and obtain new compounds for evaluation. The botanical supplement companies are finding new products from various parts of the world to market, with only a modicum of concern regarding the sustainability of their sourcing, the consistency of their constituents, and the limited scientific background justifying their use. The nutraceutical companies are looking to exploit the academic discoveries of compounds, such as cancer chemopreventive or cholesterol-lowering agents, which can be added to highvolume foods, or they are seeking plants which offer new and diverse life-style marketing opportunities (noni fruit and acai berry are recent examples). Investment in research to justify the safety, use, and quality control of these products is minimal.

With this summative background concerning the past and present practices of natural products in the discovery of new medicinal agents, it is opportune to consider the appropriate role of natural products in health care systems globally for the next twenty years.

\section{Future Aspects of Natural Products in Drug Discovery}

For future generations to thrive, be productive, and lead meaningful lives in their respective societies, there are two dominant factors to be considered, the health of the planet and the health of the people. These are not to be separated, as we are all part of one large, fully interacting, biological organism, Earth. The goal must be to maintain the health of that whole organism in a cost-effective manner. Failure to do so will leave a terrible legacy for our descendants. Consideration of all chemical processes, including those for the synthesis of medicinal agents, and the accessibility of drugs to those in need are critical. Factoring in the anticipated cost to the patient becomes an important consideration as potential drug candidates in any form, synthetic or natural, make the transition from discovery to clinical development. Already there are numerous situations where the latest drugs are becoming (or have become) too costly for health care systems, including insurance companies, and their patients in the high-income world. When it is a Health Ministry in a country which decides which drugs are to be imported, and at what cost, for their health care systems, very significant compromises are typically made in terms of access to optimal health care. In the course of time, these differences in access are clearly reflected in 
life-expectancy, and become another global pharmaceutical care "gap" between the North and the South. At the same time, it is also recognized that enhancing the quality of traditional medicines will have an impact on product costs. This is contained within the concept of accessibility, the combination of sustainability and affordability.

Almost lost now in the mists of time, it should be remembered, that before 1899 , when the semi-synthetic drug aspirin, based on the natural pain reliever salicin, was introduced, all drugs were derived from natural sources. Since then, a vast pharmaceutical industry, with many companies tracing their history to the original sellers of natural products in the latter part of the $19^{\text {th }}$ century, have evolved to develop new drugs for a myriad of diseases. Some of the compounds introduced during that period from 1900-2010 have been natural products, and the more recent discovery efforts have been reviewed by Newman and Cragg $(28,29)$. Most of the drugs that have been introduced in the past 110 years are totally synthetic. The primary exceptions are several groups of antibiotics, and the steroid and alkaloid derivatives which are semi-synthetic.

It is time now to introduce the new term "ecopharmacognosy" (38). As we look to the future for the practice of using synthetic medicinal agents, one aspect of concern which requires deliberation is the sustainability of the production of those drugs at reasonable cost. Both the chemicals and the chemical reagents used in those processes are typically a nonrenewable resource, and their contemporary use depletes the future resources for synthetic drugs. Consequently, a fundamental precept for all drug discovery programs, be they synthetic or natural, must now be the concept of sustainability $(4-12,38)$, as an extending consideration of the "green chemistry" movement. One aspect of this topic was discussed earlier in terms of the development of alternative, renewable sources for chemical reagents (enzyme catalysts, vegetables, etc.) (13). Selected, valuable traditional medicines have been, or are being, depleted in their natural environment, without alternative resourcing being developed. Ecopharmacognosy, defined as the "study of sustainable, biologically active natural resources", must therefore become the fundamental basis for all natural product research. Studying plants, or any natural organism, for their use as a global medicine, must take into account the potential long-term sourcing. We have already seen situations, taxol is an example, where the drug requirements for a clinical trial, came up against serious sourcing concerns. Consideration of the enzymes to be used for the large scale synthesis of drugs, must also consider the sustainability of the resource. Using the enzymes in plants that are already commercial entities, such as vegetables, assures their long-term accessibility, and reduces cost compared with rare enzyme preparations derived from microbial sources. Ecopharmacognosy therefore embraces both the development of biologically active natural products as single agents or as components of traditional medicines, and the resources for the chemical synthesis of single agent drugs. The same considerations must also be applied as marine resources are explored for the development of new biological agents.

Except for some biological agents, such as vaccines, which will not be discussed here, there are usually three classes of single agent drugs recognized: i) totally synthetic drugs, which are produced from non-renewable resources, such as coal and oil; ii) semisynthetic drugs, such as the steroid hormones which are derived from a chiral natural product core produced in the field, or, in the case of antibiotics, through large scale fermentation. This is followed by structural modification using nonrenewable resources, unless microbial transformations are included in the synthetic strategy; and iii) natural products, such as vincristine or morphine, which are derived from a renewable natural resource. In this instance, the non-renewable aspects involve the materials used for processing the plant material in order to isolate the desired alkaloid.

Of perhaps thirty different aspects which could be discussed regarding the future for natural products in drug discovery, six will be briefly presented: i) access to the biome, ii) acquisition and analysis of traditional knowledge and on-going research, iii) biotechnology development for secondary metabolites, iv) dereplication studies, v) strategies in natural product drug discovery, and vi) multitarget therapy and synergy research.

\section{Access to the biome}

The natural materials to be used, and any ethnomedical knowledge not already in the public domain, are the property of the country in which they are extant historically. The Convention on Biological Diversity (CBD), approved for signature at the Earth Summit in Rio de Janeiro in 1992, established those parameters for those signatories who ratify the treaty. As of April 2013, 193 countries were parties to the convention. Only three countries in the world had not ratified the convention: Andorra, the Holy See, and the United States. At the same time, there is a natural, unresolved tension with the TRIPS (Trade-Related Aspects of Intellectual Property Rights) agreement of 1994 in the area of the development of local resources by third parties. In the TRIPS agreement, the country of origin of the genetic material has no sovereign rights over their biological resources or their indigenous knowledge once there is an invention. It is the inventor who can claim the intellectual property rights, with the originating source of the material, whether it is derived from a plant, animal, or fungus in a particular country, having no claim for compensatory loss. The United States was the promulgator and the first signatory country to this Agreement. This area, and the whole topic of the impact of the CBD on natural products research, has been reviewed (39), and the discussion will not be repeated here. Some selected aspects of the CBD that relate to natural product drug discovery will be mentioned briefly. 
Article 15.2 of the CBD indicates that signatory countries should facilitate access to their biome in exchange for present and future considerations which are to be negotiated $(39,40)$. Articles 15.5 and 15.6 indicate that all of the collections of biological material which are made within a country should occur with prior informed consent, and that collection of the materials should occur with the accompaniment of local scientists. Article 16 is concerned primarily with issues related to access and the transfer of technologies relevant to the conservation and sustainable use of genetic resources under fair and favorable terms. Article 17 relates to the information concerned with the conservation and sustainable use of biological diversity and the results from scientific and socio-economic research are promoted. Article 18 promotes international technical and scientific cooperation, and the development of joint ventures.

Following the CBD, there were numerous developments related to the establishment of protocols and systems within countries for the approval and collection of biological materials, and the acquisition of indigenous knowledge (39). Indigenous groups, sometimes collaborating across national boundaries, have also sought to establish parameters and protocols to receive authority to provide access. While this has had the beneficial effect of reducing the number of scientists from various countries taking unauthorized plant materials or other biological resources out of a country without local knowledge (sometimes referred to as "biopiracy"), there has been a clear tendency towards the over-protection of access to resources, and/or excessive bureaucracy associated with the approval process (39).

It is this outcome of the CBD, doubtless not anticipated by the drafters or the signatory parties, which has led to the overall deleterious impact of the CBD on natural products research on a global basis. This is because the value placed on the access to those resources by an individual country was often too great. The expectation that pharmaceutical companies would line up to seek access to areas of intense biodiversity, never materialized. As a result, if the approval processes, or the restrictions, or the time and the financing required to negotiate the associated considerations, were deemed too onerous, academic and/or industrial laboratories declined to invest, in either the people or the places. Particularly, this occurred if more amenable choices for sample acquisition (based on bureaucracy, cost, and time), which might well have the same plant materials, were available. Alternatively, many pharmaceutical companies simply chose to eliminate natural product extracts (marine, fungal and plant) from their primary screening programs, and, at the time, turned to other sources, such as combinatorial chemistry, for the guaranteed expansion of their chemical libraries.

This resulted in two scenarios. Firstly, the biodiversity-rich countries were unable to find collaborations (either academic or industrial) for programs designed to evaluate their biome for a health care impact, and were unable to enhance the development of much-needed taxonomic, chemical, and biological capabilities in-country. In some instances (e.g. the Philippines), local scientists were also severely impacted by strict government requirements, and their research was essentially halted for extended periods (34). Secondly, local pharmaceutical development, deemed critical for the type of initiatives being discussed to improve local health care, was inhibited. As a direct result, the reliance on externally acquired pharmaceutical and medicinal agents was maintained, benefitting the major drug manufacturers. Without access to biological materials, the discovery of new biologically active natural products is diminished and advances in health care are impeded in that country and elsewhere.

It is important that prior negotiated agreements are seen as an essential aspect of plant collection programs for drug discovery, and there is a sense of pragmatism required. Very, very few compounds, or their semisynthetic derivatives, will ever become a profitable invention. An alternative pathway is to negotiate a graduated, two-tier approval process for the collection of biological materials which distinguishes between the "discovery" phase and the "development" phase. Thus, one agreement would cover initial collection and academic/pre-toxicological research on limited size samples, and the second agreement would apply only when the acquired sample size had to be increased for more advanced pharmacology and clinical studies. $(39,41)$.

On October 29, 2010, the "Nagoya Protocol on Access to Genetic Resources and the Fair and Equitable Sharing of Benefits Arising from Their Utilization to the Convention on Biological Diversity" was adopted under the auspices of the CBD (42). It is an instrument for the implementation of the access and benefit-sharing provisions of the CBD, and was opened for signatures on February 1, 2011. It was developed after six years of negotiation, and overall content supports and compliments the CBD. Only countries which have signed and ratified the CBD are eligible to sign or ratify the Nagoya Protocol, but a country can also have signed the $\mathrm{CBD}$ and choose not to sign the Nagoya Protocol. When fifty countries had signed it entered into force and countries began implementation. As of April, 2013, 92 countries had signed the Nagoya Protocol, and 16 had ratified the Protocol (43).

The primary focus of the Protocol is on the equitable sharing of benefits, and the requirements of signatory nations to develop procedures for implementation and regulation of the $\mathrm{CBD}$, with a specific requirement for the issuance of permits with respect to permission granted for access to either genetic resources or indigenous knowledge. The Protocol establishes an international Clearing House under the CBD secretariat to assist countries with respect to developing various aspects of the implementation process, and requires countries to deposit appropriate records and information from their country with the Clearing House for common availability. Specific issues relating to trans-boundary situations must be discussed in instances where indigenous groups overseeing knowledge or resources are not located in a 
single country, as may occur in parts of the Andean region, in the Middle East, and in Southeast Asia.

In response to significant criticism about bureaucratic process and openness, ratifying countries are now required to assure legal certainty, clarity and transparency, both legislatively and in the implementation of regulatory requirements, such as applications for prior informed consent. Countries must also provide effective communication systems during periods of application evaluation. Permits which are internationally recognized will be issued by the recognized national authority for approved programs in a country based on prior informed consent and mutually agreed terms. This information will be provided to the newly-established CBD Access and Benefit-sharing Clearing House. One can imagine that the permits will be needed both internally at various points in the process, at the collection site, at the exportation site for genetic material, and probably by major international journals in the field to assure compliance with international standards for published research articles.

Somewhat surprisingly, the contentious issues related to patents are not discussed in the Nagoya Protocol, even though it is an essential consideration for many groups seeking access to genetic resources and traditional knowledge, and an anticipated constituent aspect of any prior negotiated agreement between parties. Consequently, the obvious tensions with the TRIPS Agreement remain unresolved. Directly related to the concerns regarding patents and the non-obviousness of inventions is the issue of "derivatives", which the Protocol defines as "a naturally occurring biochemical compound resulting from the genetic expression or metabolism of biological or genetic resources." This is not a robust definition of a derivative, and it is easy to imagine how a corporate entity could develop derivatives, be in accordance with mandates of the Nagoya Protocol, and still be the sole recipient of patent rights. Another significant omission from the Nagoya Protocol is the absence of mandatory checkpoints or benchmarks which a certificate holder should be required to reach during the application and experimental processes. Individual countries are however, permitted to include benchmarks of performance and reporting as they deem necessary.

\section{Acquisition and analysis of traditional knowledge and on-going research}

It has been said that the countries which will be successful in science and technology in the future will be those who use the globally available knowledge most creatively to generate new knowledge and inventions. This "smart" technology is desperately needed as strategies and considerations for the exploration of the tropical forest are initiated. Information, ethnomedical, botanical, chemical, and biological, is burgeoning. New technologies which may impact drug discovery programs are being developed at an incredible pace. Almost every country, and within them most scientific research laboratories, when they are connected to the internet, have essentially unlimited access to this information, most frequently at no or minimal cost.

With respect to traditional knowledge, the ease of access to information has its benefits and hazards. The benefit is that more information on the use of a plant can be compared than merely relying on one or two local compendia of information. The hazard is whether all of the available information has been acquired legally under local regulations, particularly knowledge of the use of indigenous plants acquired since the CBD. Another consideration is that the vast amount of published ethnomedical information, collected by ethnobotanists and medical anthropologists, on the use of plants for medicinal purposes, is extremely scattered, and is therefore very difficult to acquire in toto. Undoubtedly, there is always more literature to be unearthed on the use of a particular plant, if only the resources were available.

As stated on several occasions $(3,4,6-12)$, there is a dire and urgent need for an international agency, possibly in collaboration with a global foundation, to fund the development of a central repository of indigenous knowledge, a sort of "WikiEthnoMed". But the data base repository should not end there. As well as ethnomedical information, data on the biological evaluation of plant extracts and their constituents, the chemistry of the natural plant sources, and the clinical evaluation of plant extracts, needs to be acquired and collated. Data on the safety and possible or observed adverse events associated with traditional medicines are also required to be collated for open access, as a health care consideration for practitioners and patients. At this stage in the $21^{\text {st }}$ century, it should be possible, where ever one is in the world, to indicate a plant name and then find its barcode (vide infra), ethnomedical information, chemical constituents, biological activities, and clinical evaluation data. It is estimated that such an on-line, global health resource, once established, and depending on physical location(s), would cost about $\$ 5$ to 7 million to operate each year; a veritable bargain given the health care benefits.

For the development of rational, sustainable drug discovery from plant sources the first step is a critical evaluation of all of the available information on a plant, or on the plants to be evaluated for a particular health benefit, such as antihyperglycemic activity. This is needed in order to prioritize plant acquisition plans, avoid the unnecessary duplication of research effort, and optimize the consumption of precious (financial, personnel, and oil-based) resources. There are also important ecopharmacognosy considerations of sustainability which enter into these strategies as well.

\section{Biotechnology development for secondary metabolites}

When one acquires, dries, and then extracts a plant material, the observed chemical profile is the phytochemical equivalent of a "Kodak moment" (10-12). For over 180 years, phytochemistry, and by inference chemotaxonomy, tacitly accepted that this "moment" represented the biosynthetic capacity of that plant. Now 
it is well-established, through the use of tissue culture, cell-free systems, and elicitor molecules such as methyl jasmonate, that this is an inappropriate paradigm, and represents only a partial view of genetic capacity for secondary metabolite formation. Indeed, it is more like a "still shot" from the dynamic secondary metabolite profile "movie" of the plant. For no single plant on Earth has the full metabolic profile yet been determined through analysis of the genes of secondary metabolism and then demonstrated through expression. A more complete understanding of what a plant is in terms of a chemical factory for making secondary metabolites of biological or clinical interest, may be very important to achieve on a selective basis in the future.

Thus, a single extract of a plant, taken at a single point in time, cannot reflect the constituent range of that plant, ignoring as it does numerous intrinsic and extrinsic factors, including dormant biosynthetic genes which will alter the chemical profile, and thus the biological outcome. The ability to modulate these genes, and thus the enzymes they code for, in a controllable manner, will be a critical aspect to enhancing plant secondary metabolic diversity for biological screening, and in establishing reproducible production levels for needed metabolites, especially for the constituents of traditional medicines.

Fungal genetics has progressed remarkably from the perspective of understanding secondary metabolic profiles and production in recent years, and inferences with respect to the evolutionary aspects of fungal organisms are developing rapidly (44). Because of challenges with the locations of metabolic genes, plant genetics has lagged somewhat. However, as these details of the plant genes responsible for secondary metabolite biosynthesis are recognized, it becomes possible to express the genes in other, faster-growing formats $(E$. coli, yeast, insect cultures, etc.), and produce either a new range of metabolites previously unknown from that plant, or a desired enzyme, compound or series of compounds with a significantly higher degree of control $(45,46)$. From a biological screening perspective, it will be important to identify the regulatory genes responsible for secondary metabolite production control, and thereby modulate the secondary metabolite profile, so that rather than being a snapshot, it can indeed become a movie.

Both the short and long-term implications of this are clear. Maintaining the genetic capacity of the tropical forests, which has taken billions of years to evolve, through conservation, acquisition and botanic garden development, or sampling and storing germplasm, is a critical aspect of a diversified and well-considered longterm medicinal agent discovery program for a nation. It may be that locked in the DNA of a plant are the genes to produce crucial drugs in the future, once it is possible to more completely understand the contortions of their metabolic formation. Certainly, it is unconscionable to destroy the forests without first sampling and preserving the breadth and depth of the diversity of genetic resources that are within.
There are numerous other aspects of biotechnology which impinge on natural product drug discovery and development, including bioassay design and implementation, drug delivery systems, and enhancement of natural product structural diversity (vide infra). Some aspects of these technologies have been discussed elsewhere $(5-7,9-12)$.

\section{Dereplication studies}

Plants are chemical factories which produce a wide range of metabolites of various structural types. Many plants across taxonomic borders have evolved the genes to produce similar or identical metabolites. The wide spread occurrence of common flavonoids, such as quercetin and related derivatives, is an example. Once an extract is declared "active", prioritization along with other "active" extracts for bioactivity-directed fractionation is needed. Frequently, there are more active plants than capacity (human) to fractionate. At this point, there are three research options: i) eventually fractionate all the extracts; ii) introduce a secondary bioassay to discern a more selective priority list, or iii) apply a dereplication strategy.

Dereplication is a process to delineate, in an active extract, the probability that the active principle is likely to be novel. Such a determination can re-prioritize active extracts, and/or eliminate extracts for further examination, and it can improve the efficiency of isolating the active principle. Several years ago we described an HPLC/ESMS/bioassay/database dereplication system for active natural products in biologically active matrices (47), and went on to describe how it was used on active extracts for the identification of both new and known natural products (48). Refinements in dereplication systems include: i) development of software which can rapidly correlate mass and biological data to a chemical and biological database so that searches and conclusions can be achieved on-line, ii) introduction of continuous flow NMR to support postulated structures of active masses, and iii) recognition of carbon-13 NMR to provide an even higher level of certainty regarding the possible skeletal structure and functional group disposition of a molecule within the matrix. Over time, the ability to characterize the majority of the known major active constituents in an extract without isolation will become a standard practice. A further use for such HPLC/ESMS/NMR/bioassay system technology, other than drug discovery, will be for the chemical and biological standardization, as well as metabolomic studies of traditional medicine samples, on a batch to batch basis, once the appropriate active principle(s) have been identified.

\section{Strategies in Natural Product Drug Discovery}

It is an important question to ask whether natural products are drug molecules. Several years ago (21), it was shown that those alkaloids which are presently available as pharmaceutical agents are a good fit (with some outlying molecules) to Lipinski's "rule of five". When an analysis 
of 120,000 compounds in the Dictionary of Natural Products was conducted, $65 \%$ had no violations of these rules, and this led to the development of a natural product library of over 500 compounds for drug screening (49). Other studies have supported these conclusions $(50,51)$. Thus existing natural products can form the basis of a drug discovery program complementing the development of potential new sources of bioactive molecules.

Strategic considerations for the development of natural product drug discovery programs abound $(17,19,52-54)$. How the plants will be collected (random, phytochemical, ethnomedical), how plants will be extracted (water, non-polar, and polar solvents), which biological assay to use (animal, whole cell, organ tissue, enzyme, receptor), and how to establish levels for "activity" are strategic decisions which must be taken prior to initiating the program and then reviewed regularly during the program.

It is also important to recognize that a plant is not a single organism. There are numerous fungi, bacteria, and, in some cases, algae, symbiotically associated with the plant. These organisms are also biosynthetic factories, and the study of pathogenic and parasitic organisms may offer the potential to enhance the range of natural product structures in a sustainable, albeit rather unpredictable, manner.

Accessibility to resources, reproducibility of activity in recollections, and the time to yield an active isolate are important concerns in the involvement of plant extracts in drug discovery $(1-3,10-12)$. There are several other issues which can also affect the overall discovery process. We have seen that isolation of known compounds with a known bioactivity is a significant issue in some biological areas (such as cancer), and there is also the patent concern of known compounds which may afford new biological activities. Patenting inventions has become an important, though not essential (vincristine, taxol, and camptothecin were never patented), function for both industrial and academic discovery programs, and the strongest drug patents are those which claim a new chemical entity with a new biological activity.

Sample repositories are an essential aspect of any plant-based drug discovery programs. Typically, as a program evolves, there are three types of repository that are needed: i) for the plants acquired, ii) for the extracts made, and iii) for the compounds isolated. The first may, in part, be the local, internationally recognized, herbarium where a formal, classically prepared specimen is stored for posterity. Also needed is a dry, airconditioned, moderate temperature $\left(0-10^{\circ} \mathrm{C}\right)$ facility for storing the remainder of collected, but unused samples, appropriately labeled and catalogued in a database. The second repository is for residual samples of all extracts and fractions that are prepared, usually held at $-20^{\circ} \mathrm{C}$ or below, and again systematically catalogued electronically and organized in individual, barcoded vials for easy access. Frequently, sample specimens of extracts are also stored in 96-well plates for screening purposes, depending on strategic demands based on the available and projected biological assays. Finally, purified and isolated compounds must be catalogued and stored, both in vials, and in 96-well plates for further screening. Long term, the drug discovery goal should be to screen every isolate against every relevant bioassay. Electronic cataloguing and analysis of this information is important, as is recognizing that data base access should be available to all collaborators. Of course a fourth database, not experimental in nature based on the current studies, should constitute an inventory of information on the previously reported studies on all of the plants actively under study. The first step in deciding whether to actively work on a "hit" extract is to become fully aware of all of the prior literature on that plant and related species. It is another example of "information first" as a philosophical precept.

Identification of the plant materials to be used for discovery purposes is very important as a basis for the whole program. But how is the plant to be identified? Preliminary identification may come from morphological examination and herbarium specimen comparison. For several years, DNA techniques, such as random amplified polymorphic DNA (RAPD), amplified fragment length polymorphism (AFLP), and inter-simple sequence repeat (ISSR) analysis were deployed as an adjunct for plant identification, and for studying the genetic variation in medicinal plants (55). Now the focus is on the development and application of DNA barcoding (56), as one facet of the Consortium for the Barcoding of Life (CBOL), and some background details pertaining to medicinal plant identification have been discussed (9-12). The DNA fragments which are considered appropriate to use for species identification at this time are focused on the ITS2, matK, and trnH-pasbA genes. Reliability rates, the ability to make positive medicinal plant identification based on specific genes at the genus and species level, are typically very high (76-96\%). Chen and co-workers (57), using seven DNA barcodes (psbA-trnH, matK, rbcL, rpoC1, ycf5, ITS2 and ITS), studied over 6600 plant samples from 753 genera and 4800 species. They found that the ITS2 barcode gave a $92.7 \%$ successful identification rate, and thus could potentially used for the identification of medicinal plants. How this relates to secondary metabolite production, and therefore to a reproducible biological activity, remains an area for future exploration. Another significant future development is the incorporation of barcoding technology into highly automated, hand-held devices which could provide extremely rapid, in-field identification of plants (58).

Principal component analysis (PCA) of the low molecular weight (ca. 200-500 daltons) compounds in a plant as a part of metabolomic studies is also changing how a plant is defined (59). It will become an important component of drug discovery programs to assure adequate compound sourcing in recollected materials, either for expanded biological evaluation or possibly to meet production demands. The combination of these techniques means that the identification of plants through gross morphology or macroscopic examination is rapidly being supplanted. 
The gene-based and chemical analysis techniques now demonstrate that within a recognized plant "species" there are many "forms", "races", or "chemotypes". These plants will have different functional genetic profiles under different circumstances, different biosynthetic capacities based on those gene profiles, and therefore variable and complex chemical matrices. This begins to explain why reproducible plant recollection is so challenging and frustrating. Within the next few years, DNA barcoding, and probably PCA, will become essential and integral aspects of all medicinal plant identification, whether for drug discovery programs, or for standardization of traditional medicines and phytotherapeuticals. The result may be that the concept of a Latin binomial name for a plant, romantic though that is, will be replaced, or added to, by a series of identifiers which will define both the integrity and the quality of the sample.

In the biological evaluation of plant extracts tannins are a frequent confounding factor and must be removed when either enzyme or receptor-based assays are involved (60). This has led to the development of detannification and partial fractionation techniques to form peak libraries of dominant compounds in an extract which can then be screened. While minor bioactive compounds may be missed, this strategy does begin to address the issue of adequate subsequent supply when bioactivity is found, a core consideration of ecopharmacognosy (38).

Various data mining strategies may also be used to construct libraries of plant extracts or individual compounds, once adequate, pre-existing literature has been compiled. Chemical space matches and pharmacophoric models also provide an in silico approach to the identification of compounds of potential interest which can then be isolated or further derivatives sought. Rapid access to plant materials as potential resources, based on existing isolation or chemotaxonomic information to identify plants for collection, is then an essential component of the discovery strategy.

\section{Multitarget Therapy and Synergy}

In considering both the quality control and the drug discovery perspectives of traditional medicines, two aspects are significantly undervalued in terms of therapeutic outcome, multitarget therapy and synergy. Rather than the classical "magic bullet" approach, therapeutic outcomes have improved significantly for two of the most important disease states, cancer and AIDS, as a result of strategically assembled multicomponent regimens derived from considering a diverse mechanistic targeting system (61). Ethnomedicine has already established that model of therapy. The chemical factory of a plant, and even a hot water extract, will contain a multitude of constituents, and multicomponent plant regimens, in which five, ten, or even twenty plants are used in a prescription, provide an unprecedented range of highly diverse chemical constituents which are affecting multiple sites and acting by diverse mechanistic pathways. An important question is whether there is clear evidence of rational use for the role of each individual component plant in a multicomponent traditional medicine? As evidence-based traditional medicines are explored for drug discovery and validation this will become an important experimental target, and eventually a consideration in the sustainability of that product. A recent discussion on network pharmacology provides an important framework for these deliberations, and for the potential design and discovery of new agents with unsuspected biological activity (62).

The biological effects observed in vivo or clinically in an extract may be due to one or more active compounds acting at different sites. Alternatively, two, or more, components in the mixture could be acting in a synergistic or in an antagonistic manner. In addition, the effects when a traditional medicine is taken with a single agent drug are unknown, resulting in a possible adverse drug reaction (ADR) which may potentiate or inhibit the actions of the single agent drug. When combinations of medicinal plants are used, as in many drug systems around the world, the situation becomes significantly more complex. Williamson (63) and Wagner $(64,65)$ have stimulated discussion in this area.

A significant issue in studying synergy and antagonism in multicomponent traditional medicines has been technique and definition (65). Berenbaum (66) used a mathematical definition based on an isobole to represent these biological outcomes, so that the effects of a combination of agents are independent of the mechanism of action, and can be presented graphically. A powerful demonstration of a synergistic interaction between two natural products occurs with mixtures of ginkgolides A and $\mathrm{B}$ examining platelet aggregation (67). Potentiation of the effects of kava-kava and a Passiflora extract as a sedative, and of a complex preparation of nine plants for dyspepsia whose constituent plants demonstrate effects on a range of motility-related disorders have also shown synergistic results $(64,65)$. The protocols developed for these studies may have an important influence on the evolving strategies for the evaluation of the quality, safety, and effectiveness of an individual traditional medicine, and on the discovery and development of new, more effective combinations of medicinal plants ("designer traditional medicines"). In addition, they may offer strategies to increase the sustainability of particular medicinal plants, if the synergistic effects can be quantified and reliably reproduced. Critically, such experiments require that the extract is well standardized and the biological mechanisms are well clarified prior to synergy experiments being initiated.

\section{Challenges and Strategies for the Future}

A vision for natural products derived from the tropical forest is based on the concept that sustainable considerations and strategic thinking are needed now. In addition, those resources will be needed even more in the future, in order to provide medicinal agents as fossil 
based resources become depleted during a period when the global population is increasing rapidly. Such a vision involves the creation of a new term, ecopharmacognosy, to describe the philosophical shift, and requires new paradigms for the conduct of the natural product sciences on a global basis, including new balances of program development for drug discovery and traditional medicine quality control, new strategies, a new emphasis on evidence-based research, new alliances between various collaborators in academia, industry and government, and new values for what is meaningful, natural product research for health care enhancement $(8-12,38)$.

It should be readily apparent that humankind cannot survive another century as destructive of the Earths' resources as the $20^{\text {th }}$ century. Twenty-one years after the Rio Summit of 1992 we are as "irresponsible" as ever with respect to our biodiversity. At the end of the last century one-eighth of all plant species were threatened, $50 \%$ of bird species are likely to become extinct by 2050 , and affordable oil resources are estimated to last until 2060. The abiding concern which the world must grapple with now is whether, as a human race, we can see these issues as being our destruction and own our responsibility in any attempted restoration of a balance within Gaia (l).

It is critical that we are mindful of the balance between the conservation of the existing rain forests, and the destruction of these fragile and deeply interwoven ecosystems and their deforestation for crop and grazing lands. Our concerns are for ecological, climactic, and geological reasons, and as a way to maintain the bio-, and therefore chemo-, diversity within those forests. As described within ecopharmacognosy, plans for the development of new medicinal plants in any form to fill a market niche must be sustainable. An appropriate balance is needed between intellectual property rights and the burgeoning technology of drug discovery. A balance is also needed between all those who are stake holders for biodiversity and indigenous knowledge and those who have the capacity to potentiate (create value) in that biodiversity for health and economic benefit. This is the balance that is needed between the CBD/Nagoya protocols and the TRIPS agreement (39).

Numerous new alliances, both internal and external will be needed to effect the rational development of tropical forests for new medicinal and biological agents. Some may already be in place; they need to be strengthened. Other alliances will be new; they will need to be carefully nurtured. Diversity of expertise and experience indicates that the alliances will be both local and global in nature. They will involve individuals and groups who have the capacity for high level collaboration and low level ego involvement. Several examples of such collaborative programs have been described (1,52-54). The strongest programs, rather than being solely based in academia, will have industrial and government partners working together for a common goal, enhanced health care for the patient. Countries which are engaged in medicinal agent discovery programs based on local natural resources are recommended to examine the structure and functioning of these programs as a potential model for collaborative investment to promote the development of local medicinal plants and natural products. In a sound and comprehensive health care program, such alliances are an integral aspect $(6-12,38)$. In order to accomplish these goals though we must create value; value in places, in people, and in plants.

The tropical forest is a huge vast ecosystem which is comprised mostly of "weeds", those "plants whose virtues have yet to be discovered", as Ralph Waldo Emerson suggested in the late $19^{\text {th }}$ century. An important responsibility for natural product scientists to the generations following is either to demonstrate the value of biodiversity through new discoveries of plant-derived medicinal agents, or to leave the resource alone and protected. Solid linkages which unite the interests of environmental preservation, medicinal plant research and drug discovery, and the development of the agroindustrial enterprise are therefore crucial. Examining import levels of finished pharmaceuticals and natural products (essential oils, and flavor and fragrance materials) in the context of developing local industrial capacity is important. Structured and well-monitored natural product development programs based on tropical forest resources could lead to reduced imports and increased exports.

A vision of the natural product sciences contributing to global health care offers very significant challenges to many countries. A primary challenge is to catalog and preserve the bio- and chemo-diversity of the rainforests (and the oceans) for the benefit of future generations. A secondary challenge is the need to catalog the eco- and ethno-information on plants, and the chemistry and biology of their products, so that the information can be collated, analyzed, and accessed globally in real time.

Exploration aimed at potentiation of the biota of the world from any of several aspects, including drug discovery, necessitates systems being available locally which can offer equitable access to the biome and substantial assurances with respect to intellectual property rights and investment commitments. Many medicinal plants, some of them threatened or endangered, are presently in commerce in various parts of the world, either for the production of single agent drugs, or in traditional medicine preparations. Medicinal plant germplasm banks in selected locations throughout the world are needed to preserve these crucial resources, in the same way that seed crop banks are used for important food crops. At the same time, contemporary techniques for plant identification, including DNA barcoding, must become integral aspects of what is considered plant identification, whether these plants are being investigated or used as medicinal plants, or whether they are being pursued for possible drug discovery of standardized preparations or single agents.

The sustainable development of plants and their extracts or fractions which are marketed for health care purposes is critical. A "Sustainability Index" probably needs to be developed, so that patients can clearly see from a label how the product was harvested and prepared. A product derived directly from the forest and extracted 
in a manner where an organic solvent was not fully recycled would get a rating of " 0 ", whereas one harvested organically in a sustainable manner with full recycling of any solvents, might get a rating of " 5 ". Individual compounds and plant extracts must be evaluated using contemporary procedures and targets for both quality control and drug discovery. Strategies should be developed for genomics-based, in-field bioassays to evaluate plant extracts in the field, to avoid bringing dried (or fresh) plant materials to the laboratory for extraction and bioassay. The number of natural products presented to test systems for biological evaluation could be enhanced through chemistry, combinatorial synthesis, combinatorial biosynthesis, and other strategies. Drug discovery programs must be introduced, strategically developed, and enhanced based on local plants and for local diseases. Most importantly, integrated global alliances are needed both in-country, and between developed and developing countries, for medicinal plant product development, in order to optimize the utilization of facilities, infrastructure, and personnel who are needed to conduct the above programs.

How can these goals and the associated programs be initiated? First of all it must be said that plans of the scale described in this article and elsewhere (4-12,38) begin with small scale initiatives, reasonable benchmarks, and an appropriate timetable. They are not instantaneous "fixes" of the situation, nor will they yield rapid results. Strategic planning at the highest levels is required, combining government agencies, industrial enterprises, international agencies and foundations, academic institutions, and private consultants with natural product drug discovery and development experience. Planning and proposal development may take 1-2 years of meetings, discussions, and consultations to evolve. Over time, countries, or consortia of countries, will require an infrastructure to foster the development of their own sustainable medicinal agents from natural sources based on the quality of their natural product sciences. Programs will be needed to assist countries to potentiate their infrastructure and resources, including their facilities and their scientists, in order to evaluate and standardize natural product-based medicinal agents on a sustainable basis for their health care systems, and that may take 510 years to evolve.

Ideally, as proposed originally in 2002 (3), what is drastically needed is a Global Alliance for Natural Product Development and Health Care. Such an alliance would be composed of international agencies (WHO, UNIDO, UNDP, NATO, EU, WIPO, etc.), government agencies (NIH, NSF, NIE, SRC, DAAD, etc.), global and local pharmaceutical companies, academic institutions, non-government organizations (WWF, WRI, CYTED, TRAMIL, IFS, TWAS, etc.), scientific societies (IUPAC, RSC, ASP, PSE, GA, JSPS, etc.), and major foundations (Ford, Gates, MacArthur, Rockefeller, Nippon, etc.). A mechanism to bring representatives together to discuss the global issues and implications in new terms, with a new set of goals, with a new agenda, but most importantly with a new vigor, is vital for the global development of natural product based drugs for health care.

\section{Conclusion}

Globally, natural products, in the form of purified active principles and plant extracts, are the cornerstone of primary health and for the amelioration of life-style conditions, and will remain so for decades to come. With a burgeoning population, the challenges for health care in the future remain significant. For most of the major killer and chronic diseases in the world there are no truly effective drug treatments. Drug resistance to existing chemotherapeutic regimens for fungal and bacterial infections, for AIDS, for cancer, and for malaria, is increasing in an unabated and alarming manner. Many killer and debilitating diseases in the middle and lowincome areas of the world exist without any effective drug treatment, or even a drug discovery program, in place. Yet, overall health care is slowly improving, life expectancy is rising in most countries, and more children are surviving beyond their first five years. These are contributing factors to the dramatic rise in the global population. However, without a new strategic approach, this population will not have adequate resources to provide basic health care. There is no choice in this matter, medicinal plants must be an essential, sustainable, and fully integrated element in any global health care strategy.

Essential components of a strategic plan for potentiating the rain forest for health purposes include: enhancing the natural product plant sciences on an international basis, developing multi-centered research partnerships embracing the chemical and biological sciences, and expanding facilities and training programs which focus on the technologies for developing local natural products for health care, and protecting global resources. This paper, like its several predecessors (1$12,38)$, is another clarion call to critical decision-making and action by governments, international agencies, and pharmaceutical companies to commit to the sustainable development of natural products as necessary medicinal agents for an unprecedented level of human habitation of Earth.

\section{References}

Argyriou, A.A. and H.P. Kalofonos. 2009. Molecularly targeted therapies for malignant gliomas. Mol. Med. 15, 115-122.

Berenbaum, M. 1999. What is synergy? Pharmacol. Rev. 41, 93141.

Butler, M.S. 2008. Natural products to drugs: natural productderived compounds in clinical trials. Nat. Prod. Rep. 25, 475 516.

CBOL Plant Working Group. 2009. A DNA barcode for land plants. Proc. Natl. Acad. Sci. 106, 12794-12797.

Chen, S., H. Yao, J. Han, C. Liu, J. Song, L. Shi, Y. Zhu, X. Ma, T. Gao, X. Pang, K. Luo, Y. Li, X. Li, X. Jia, Y. Lin, and C. Leon 2010. Validation of the ITS2 region as a novel DNA 
barcode for identifying medicinal plant species. PLoS One $\mathbf{5}$, e8613.

Cordell, ed., Academic Press, San Diego, California, 50, 257-316.

Cordell, G.A. 1987. Pharmacognosy: far from dead, Amer. Drug. March, 96-98, 1987.

Cordell, G.A. 1990. Pharmacognosy - a high tech pharmaceutical science. Pharmacia 30, 169-181.

Cordell, G.A., C.W.W. Beecher, and J.M. Pezzuto.1991. Can ethnopharmacology contribute to the development of new anticancer drugs? J. Ethnopharmacol. 32, 117-133.

Cordell, G.A. 1993. Pharmacognosy - new roots for an old science". In: Studies in Natural Products Chemistry, Volume 13. Bioactive Natural Products (Part A). Atta-ur-Rahman and F.Z. Basha, eds. Elsevier Science Publishers, Amsterdam, pp. 629-675.

Cordell, G.A. 1995. Changing strategies in natural products chemistry. Phytochemistry 40, 1585-1612.

Cordell, G.A., M.L. Quinn-Beattie, and N.R. Farnsworth. 2005. The potential of alkaloids in drug discovery. Phytother. Res. 15, 183-205.

Cordell, G.A. 2000. Biodiversity and drug discovery - a symbiotic relationship. Phytochemistry 55, 463-480.

Cordell, G.A. 2002. Natural products in drug discovery - creating a new vision. Phytochem. Revs. 1, 261-273.

Cordell, G.A. 2002. Discovering our gifts from nature, now and in the future. Part I. Rev. Quim. 16, 5-12.

Cordell, G.A. 2003. Discovering our gifts from nature, now and in the future. Part II. Rev. Quim. 17, 1-15.

Cordell, G.A. 2004. Accessing our gifts from nature, now and in the future. Part III. Rev. Quim. 19, 33-41.

Cordell, G.A. 2004. Some thoughts on the standardization of traditional medicines. Thai J. Health Res. 18, 87-105.

Cordell, G.A. 2004. Plants in drug discovery - creating a new vision. In: Novel Compounds from Natural Products in the New Millennium. B.H. Tan, B.H. Bay, and Y.-Z. Zhu, eds. World Scientific Publishing, Singapore, pp. 1-19.

Cordell, G.A. 2005. What's in a name? And why is that important for the future of health care? In: "Proceedings of the 2nd International Conference and Exposition on the Modernization of Traditional Chinese Medicine", Chengdu, People's Republic of China, pp. 405-418.

Cordell, G.A. and M.D. Colvard. 2005. Some thoughts on the future of ethnopharmacology. J. Ethnopharmacol. 100, 5-14.

Cordell, G.A. and M.D. Colvard. 2007. Natural products in a world out-of-balance. Arkivoc, vii, 97-115.

Cordell, G.A. 2008. Natural products research - a view through the looking glass. Sci. Cult. 74, 11-16.

Cordell, G.A. 2009. Sustainable drugs and global health care. Quim. Nova 32, 1356-1364.

Cordell, G.A. 2011. Plant medicines key to global health. Chem. \& Eng. News June 27, 52-56.

Cordell, G.A. 2011. Sustainable medicines and global health care. Planta Med. 77, 1129-1138.

Cordell, G.A. 2011. Phytochemistry and traditional medicine - a revolution in process. Phytochemistry Lett. 4, 391-398 (2011).

Cordell, G.A. and M.D. Colvard. 2012. Natural products and traditional medicine - turning on a paradigm. J. Nat. Prod., 75, 514-525

Cordell, G.A. 2012.New strategies in traditional medicine, In "Medicinal Plants: Diversity and Drugs", eds. M. Rai, G.A. Cordell, J.L. Martinez, M. Marinoff, and L. Rastrelli. CRC Press, Boca Raton, FL, pp. 1-45.

Cordell, G.A., T.L.G. Lemos, F.J.Q. Monte, and M.C. de Mattos. 2007. Vegetables as chemical reagents. J. Nat. Prod. 70, 478492.

Cordell, G.A., C.W.W. Beecher, A.D. Kinghorn, J.M. Pezzuto, H.L. Constant, L. Fang, E.-K. Seo, L. Long, B.-L. Cui, and K.S. Barrilos. 1997. The dereplication of natural products. In: Studies in Natural Products Chemistry. Atta-ur-Rahman, ed.
Elsevier Science Publishers, Amsterdam, The Netherlands, 19 749-791.

Cordell, G.A. and Y.G. Shin. 1999. Finding the needle in the haystack. The dereplication of natural product extracts. Pure Appl. Chem. 71, 1089-1094.

Cordell, G.A. "Ecopharmacognosy and the Globalization of Traditional Medicine", in "Proceedings of the $7^{\text {th }}$ International Symposium of the International Society for the Development of Natural Products", Noida, Delhi, India, ed. P. Pushpangadan and V. George, 2013, In press.

Cordell, G.A. 2010. The Convention on Biological Diversity and its impact on natural product research. In: "Natural Product Chemistry for Drug Discovery". A.D. Buss and M.S. Butler, eds. Royal Society of Chemistry Publishing, Cambridge, England. 81-139.

Farnsworth, N.R., O. Akerele, A.S. Bingel, D.D. Soejarto, and Z. Guo. 1985. Medicinal plants in therapy. Bull. WHO 63, 965981.

Feher, M. and J.M. Schmidt. 2003. Fuzzy clustering as a means of selecting representative conformers and molecular alignments. J. Chem. Inf. Comput. Sci. 43, 218-227.

Ganesan, A. 2008. The impact of natural products upon modern drug discovery. Curr. Opin. Chem. Biol. 12, 306-317.

Gesell, A., M.L. Díaz Chávez, R. Kramell, M. Piotrowski, P. Macheroux, and T.M. Kutchan. 2011. Heterologous expression of two FAD-dependent oxydases with $(S)$ tetrahydroprotoberberine oxidase activity from Argemone mexicana and Berberis wilsoniae in insect cells. Planta, doi:10.1007/s00425-011-1357-00424.

Harvey, D.L. 2008. Natural products in drug discovery. Drug Disc. Today 13, 894-901.

Heubl, G. 2010. New aspects of DNA-based authentication of Chinese medicinal plants by molecular biological techniques. Planta Med. 76, 1963-1974.

Jarvis, L.M. 2010. Research recalibrated. Chem. \& Eng. News June 7, $13-18$.

Jarvis, L. 2013. AstraZeneca's new look. Chem. \& Eng. News March 25, 9 .

Kinghorn, A.D., N.R. Farnsworth, D.D. Soejarto, G.A. Cordell, J.M. Pezzuto, G.O. Udeani, M.C. Wani, M.E. Wall, H.A. Navarro, R.A. Kramer, A.T. Menendez, C.R. Fairchild, K.E. Lane, S. Forenza, D.M. Vyas, K.S. Lam, and Y.-Z. Shu. 1999. Novel strategies for the discovery of plant-derived anticancer agents. Pure Appl. Chem. 71, 1611-1618

Kinghorn, A.D., N.R. Farnsworth, D.D. Soejarto, G.A. Cordell, S.M. Swanson, M.C. Wani, M.E. Wall, N.C. Oberlies, D.J. Kroll, R.A. Kramer, W.C. Rose, G.D. Vite, C.R. Fairchild, R.W. Peterson, and R. Wild. 2003. Novel strategies for the discovery of plant-derived anticancer agents. Pharmaceut. Biol., 41 (Suppl.), 53-67.

Kingston, D.G.I. 2010. Modern natural product drug discovery and its relevance to biodiversity conservation. J. Nat. Prod., 74, 496-511.

Kutchan, T.M. 1998. Molecular genetics of plant alkaloid biosynthesis. In: The Alkaloids, Chemistry and Biology. G.A.

Medicins Sans Frontieres. Campaign for Access to Essential Medicines. www.accessmed.org/no cache/main/medicalinnovation. Accessed on August 15, $201 \overline{1}$.

McCluney, W.R. 2004. Humanity's environmental future. Making sense in a troubled world. SunPine Press, Cape Canaveral, FL. 408 pp.

Nett, M., H. Ikeda and B.S. Moore. 2009. Genomic basis for natural product biodiversity in the actinomycetes. Nat. Prod. Rep. 26, 1362-1384.

Newman, D.J. and G.M. Cragg. 2010. Natural products as drugs and leads to drugs: the historical perspective. In: "Natural Product Chemistry for Drug Discovery". A.D. Buss and M.S. Butler, eds. Royal Society of Chemistry Publishing, Cambridge, England, pp. 3-27. 
Newman, D.J. and G.M. Cragg. 2012. Natural products as sources of new drugs over the 30 years from 1981-2010. J. Nat. Prod. 75, 311-335.

Newmaster, S. G., S. Ragupathy, and J. Janovec. 2009. A botanical renaissance: state-of-the-art DNA barcoding facilitates an automated identification technology system for plants. Int. J. Comput. Applic. Technol. 35, 50-60.

Okada T, F.M. Afendi, M. Altaf-ul-Amin, H. Takahashi, K. Nakamura, and S. Kanaya. 2010. Metabolomics of medicinal plants: the importance of multivariate analysis of analytical chemistry data. Curr. Comput. Aided Drug Des. 6, 179-196.

Quinn, R.J., A.R. Carroll, N.B. Pham, P. Baron, M.E. Suraweera, G.K. Pierens, and S. Muresan. 2008. Developing a drug-like natural product library. J. Nat. Prod. 71, 464-468.

Raskin, I., D.M. Ribnicky, S. Komarnytsky, N. Ilic, A. Poulev, N. Borisjuk, A. Brinker, D.A. Moreno, C. Ripoll, N. Yakoby, J.M. O'Neal, T. Cornwell, I. Pastor, and B. Fridlander. 2002. Plants and human health in the twenty-first century. Trends Biotechnol. 20, 522-531.

Reid, W.V., A. Sittenfeld, S.A. Laird, D.H. Janzen, C.A. Meyer, M.A. Gollin, R. Gámez, and C. Juma. 1993. Biodiversity Prospecting. World Resources Institute, Baltimore, Maryland. pp. 341.

Schweiker, W. 2004. Theological ethics and global dynamics: in the name of many worlds. Blackwell Publishing, Oxford, England. $239 \mathrm{pp}$.

Soejarto, D.D., J.A. Tarzian-Sorenson, C. Gyllenhaal, G.A. Cordell, N.R. Farnsworth, A.D. Kinghorn, and J.M. Pezzuto. 2002. The evolution of the University of Illinois= policy of benefit-sharing in research on natural products. In: J.R. Stepp, F.S. Wundham, and R. Zarger, eds. Ethnobiology and Biocultural Diversity. University of Georgia Press, Athens,
GA, p. 21-30. The text is available at http://www.cbd.int/abs/text/ accessed on August 15, 2011. Information at: http://www.cbd.int/abs/nagoyaprotocol/signatories/default.shtml. Accessed on April 13, 2013.

Soejarto, D.D. and N.R. Farnsworth. 1989. Tropical rain forests potential source of new drugs? Persp. Biol. Med. 32, 244-256.

S. Li and B. Zhang. 2013. Traditional Chinese medicine network pharmacology: theory, methodology and application. Chin. J. Nat. Med. 11, 110-120.

Wagner, H. 2006. Multitarget therapy - the future of treatment for more than just functional dyspepsia. Phytomedicine 13, 122129.

Wagner, H. and G. Ulrich-Merzenich. 2009. Synergy research: approaching a new generation of phytopharmaceuticals. Phytomedicine 16, 97-110.

Wagner, H. and B. Steinke. 2005. Natural products chemistry and phytomedicine in the $21^{\text {st }}$ century: new developments and challenges. Pure Appl. Chem. 77, 1-6.

Wall, M.E., M.C. Wani, D.M. Brown, F. Fullas, J.B. Oswald, F.F. Josephson, N.M. Thornton, J.M. Pezzuto, C.W.W. Beecher, N.R. Farnsworth, G.A. Cordell, and A.D. Kinghorn. 1996. Effect of tannins on screening of plant extracts for enzyme inhibitory activity and techniques for their removal. Phytomedicine 3, 281-285.

Wetzel, S., A. Schuffenhauer, S. Rogo, P. Ertl, and H. Waldmann. 2007. Chemoinformatic analysis of natural products and their chemical space. Chimia 61, 355-360.

Williamson, E.M. 2001. Synergy and other interactions in phytomedicines. Phytomedicine 8, 401-409. 